\title{
Functional renormalization group approach and gauge dependence in gravity theories
}

\author{
Vítor F. Barra $\oplus^{1,{ }^{*}}$ Peter M. Lavrov, ${ }^{2,3, \dagger}$ Eduardo Antonio dos Reis, ${ }^{1, \$}$ \\ Tibério de Paula Netto, ${ }^{4, \S}$ and Ilya L. Shapiro $\circledast^{1,2,3, \|}$ \\ ${ }^{1}$ Departamento de Física, ICE, Universidade Federal de Juiz de Fora, \\ 36036-330 Juiz de Fora, MG, Brazil \\ ${ }^{2}$ Department of Theoretical Physics, Tomsk State Pedagogical University, 634061 Tomsk, Russia \\ ${ }^{3}$ National Research Tomsk State University, 634050 Tomsk, Russia \\ ${ }^{4}$ Departament of Physics, Southern University of Science and Technology, 518055 Shenzhen, China
}

(Received 21 October 2019; accepted 11 February 2020; published 4 March 2020)

\begin{abstract}
We investigate the gauge symmetry and gauge-fixing dependence properties of the effective average action for quantum gravity models of general form. Using the background field formalism and the standard Becchi-Rouet-Stora-Tyutin (BRST)-based arguments, one can establish the special class of regulator functions that preserves the background field symmetry of the effective average action. Unfortunately, regardless if the gauge symmetry is preserved at the quantum level, the noninvariance of the regulator action under the global BRST transformations leads to the gauge-fixing dependence even under the use of the on-shell conditions.
\end{abstract}

DOI: 10.1103/PhysRevD.101.065001

\section{INTRODUCTION}

The interest in the nonperturbative formulation in quantum gravity has two strong motivations. First, there are long-standing expectations that even the perturbatively nonrenormalizable models such as the simplest quantum gravity based on general relativity may be quantum mechanically consistent due to the asymptotic safety scenario [1] (see $[2,3]$ for comprehensive reviews). On the other hand, there is a possibility that the nonperturbative effects may provide unitarity in the fourth derivative theory by transforming the massive unphysical pole, which spoils the spectrum of this renormalizable theory [4]. The presence of such a massive ghost violates the stability of classical solutions (see e.g., discussion and further references in [5]). At the quantum level, the perturbative information is insufficient to conclude whether in the dressed propagator the ghost pole does transform into a nonoffensive pair of complex conjugate poles [6].

\footnotetext{
*vitorbarra@ice.ufjf.br

†lavrov@tspu.edu.ru

*eareis@ fisica.ufjf.br

\$tiberio@sustech.edu.cn

"shapiro@fisica.ufjf.br
}

Published by the American Physical Society under the terms of the Creative Commons Attribution 4.0 International license. Further distribution of this work must maintain attribution to the author(s) and the published article's title, journal citation, and DOI. Funded by SCOAP.
The perturbative renormalization group in this model is well explored [7-9], but it is not conclusive for the discussion of the dressed propagator. In general, the attempts to explore this possibility in the semiclassical and perturbative quantum level $[10,11]$ has been proved nonconclusive [12], and hence the main hope is related to the nonperturbative calculations in the framework of the functional renormalization group approach [13] (see [14] for an extensive review).

Thus, in both cases the consistency of the results obtained within the functional renormalization group approach is of the utmost importance. In this respect there are two main dangers. For the quantum gravity models based on general relativity, the running of a Newton constant in four-dimensional spacetime is always obtained on the basis of quadratic divergences. These divergences are known to have strong regularization dependence. In particular, they are absent in dimensional regularization and can be freely modified in all known cutoff schemes by changing the regularization parameter. This part of the problem does not exist for the functional renormalization group applied to the fourth derivative quantum gravity. However, in this case there is yet another serious problem, related to the gauge-fixing dependence of the effective average action. This problem is the subject of the present work. In previous publications [15-17] we explored the gauge-fixing dependence in Yang-Mills theories and it was shown that such dependence for the effective average action does not vanish on-shell, except maybe in the fixed point where this object becomes identical with the usual 
effective action in quantum field theory. Except this special point there is uncontrollable dependence on the set of arbitrary gauge-fixing parameters, and thus one can expect a strong arbitrariness in the renormalization group flows which lead to the fixed point and, in fact, define its position and proper existence. The main purpose of the present work is to extend this conclusion to quantum gravity. It is remarkable that one can complete this task for the quantum gravity theory of an arbitrary form, without using the concrete form of the action. One can use this consideration, e.g., for the superrenormalizable models of quantum gravity, when the perturbative renormalization group may be exact and, moreover, completely independent on the gauge fixing $[18,19]$. This example is somehow the most explicit one, since it shows that the transition from standard quantum field theory to the functional renormalization group (FRG) may actually spoil the "perfect" situation, namely exact and universal renormalization group flow.

In Yang-Mills theories [20], the gauge symmetry of the initial action is broken on a quantum level due to the gaugefixing procedure in the process of quantization. In turn, the effective potential depends on gauge [21-24]. This dependence occurs in a special way, such that it disappears on shell $[25,26]$, which means that it is possible to give a physical interpretation to the results obtained at the quantum level.

One of the well-developed nonperturbative methods in quantum field theory to study quantum properties of physical models beyond the perturbation theory is the FRG approach $[27,28]$ (see also the review papers [29-34]). As we have already mentioned above, when applied to gauge theories, this method leads to obstacles related to the on-shell gauge dependence of the effective average action.

There are some efforts to solve this problems. One of them consists from reformulation of Yang-Mills theory with the application of a gauge-invariant cutoff dependent regulator function that is introduced as a covariant form factor into the action of Yang-Mills fields, which leads to an invariant regulator action $[35,36]$. As a consequence, the effective average action is gauge invariant on shell. A second approach [37,38] is based on the use of the Vilkovisky-DeWitt covariant effective action [39,40]. This technique provides gauge independence even off shell, but it introduce other types of ambiguities. An alternative formulation was presented in [15]; it consists of an alternative way of introducing the regulator function as a composite operator. When applied in gauge theories, this approach leads to the on-shell gauge invariance of the effective average action.

In the present work, we apply the background field method [41-43] (recent advances for the gauge theories can be found in [16,44-49] and discussion for the quantum gravities case in [50]) in the FRG approach as a reformulation to the quantization procedure for quantum gravity theories to study the gauge dependence problem in this kind of theory. This method allows us to work with the effective action which is invariant under the gauge transformations of the background fields.

Despite the numerous aspects of quantum properties successfully studied with the background field method [51-60], the gauge dependence problem remains important [16] and needs to be considered in more details. We obtain restrictions on the tensor structure of the regulator functions which allows us to construct a gauge invariant effective average action. Nevertheless, the effective average action remains dependent of the gauge choice at on-shell level.

The paper is organized as follows. In Sec. II we introduce general considerations of quantum gravity theories through the background field method. In Sec. III we consider the FRG approach for quantum gravity theories and find conditions that we must impose in the regulator functions to maintain the gauge invariance of background effective average action. Based on this, we also present some interesting candidates to the regulator functions. In Sec. IV we prove the gauge dependence of vacuum functional for the model under consideration. Finally, our conclusions and remarks are presented in Sec. V.

In the paper, DeWitt notations [61] are used. The short notation for integration in $D$ dimension is $\int d^{D} x=\int d x$. All the derivatives with respect to fields are left derivative unless otherwise stated. The Grassmann parity of a quantity $A$ is denoted as $\varepsilon(A)$.

\section{QUANTUM GRAVITY IN THE BACKGROUND FIELD FORMALISM}

Let us consider an arbitrary action $S_{0}=S_{0}(g)$, where $g=g_{\alpha \beta}(x)$ is the metric tensor of an arbitrary Riemann manifold. We assume that the action is invariant under general coordinates transformations

$$
x^{\mu} \rightarrow x^{\mu}=x^{\mu}\left(x^{\prime}\right),
$$

which leads to the metric transformations

$$
g_{\alpha \beta}^{\prime}\left(x^{\prime}\right)=g_{\mu \nu}(x) \frac{\partial x^{\mu}}{\partial x^{\prime \alpha}} \frac{\partial x^{\nu}}{\partial x^{\prime \beta}}
$$

and consider the infinitesimal form of these transformations, $x^{\prime \sigma}=x^{\sigma}+\xi^{\sigma}(x)$, when

$\delta g_{\alpha \beta}=-\xi^{\sigma}(x) \partial_{\sigma} g_{\alpha \beta}(x)-g_{\alpha \sigma}(x) \partial_{\beta} \xi^{\sigma}(x)-g_{\sigma \beta}(x) \partial_{\alpha} \xi^{\sigma}(x)$.

The diffeomorphism (3) can be considered as the gauge transformation for $g_{\alpha \beta}(x)$

$$
\delta g_{\alpha \beta}(x)=\int d y R_{\alpha \beta \sigma}(x, y ; g) \xi^{\sigma}(y),
$$


where

$$
\begin{aligned}
R_{\alpha \beta \sigma}(x, y ; g)= & -\delta(x-y) \partial_{\sigma} g_{\alpha \beta}(x)-g_{\alpha \sigma}(x) \partial_{\beta} \delta(x-y) \\
& -g_{\sigma \beta}(x) \partial_{\alpha} \delta(x-y)
\end{aligned}
$$

are the generators of the gauge transformations of the metric tensor, and $\xi^{\sigma}(y)$ are the gauge parameters. The algebra of generators is closed, namely

$$
\begin{aligned}
& \int d u\left[\frac{\delta R_{\alpha \beta \sigma}(x, y ; g)}{\delta g_{\mu \nu}(u)} R_{\mu \nu \gamma}(u, z ; g)-\frac{\delta R_{\alpha \beta \gamma}(x, z ; g)}{\delta g_{\mu \nu}(u)} R_{\mu \nu \sigma}(u, y ; g)\right] \\
& =-\int d u R_{\alpha \beta \lambda}(x, u ; g) F_{\sigma \gamma}^{\lambda}(u, y, z)
\end{aligned}
$$

where

$$
F_{\mu \nu}^{\lambda}(x, y, z)=\delta(x-y) \delta_{\nu}^{\lambda} \partial_{\mu} \delta(x-z)-\delta(x-z) \delta_{\mu}^{\lambda} \partial_{\nu} \delta(x-y),
$$

$$
\text { with } F_{\mu \nu}^{\lambda}(x, y, z)=-F_{\nu \mu}^{\lambda}(x, z, y)
$$

are structure functions of the algebra which does not depend on the metric tensor. Let us stress that the mentioned features are valid for an arbitrary action of gravity, since the algebra presented above is independent on the initial action. Therefore, any theory of gravity is a gauge theory and the structure functions are independent of the fields, that means quantum gravity is similar to the Yang-Mills theory.

A useful procedure to quantize gauge theories is the socalled background field formalism. In what follows, we shall perform the quantization of gravity on an arbitrary external background metric $\bar{g}_{\alpha \beta}(x)$. The standard references on the background field formalism in quantum field theory are [41-43] (see also recent advances for the gauge theories in [16] and [48] for the discussion of quantum gravity).

In the background field formalism, the metric tensor $g_{\alpha \beta}(x)$ is replaced by the sum

$$
g_{\alpha \beta}(x)=\bar{g}_{\alpha \beta}(x)+h_{\alpha \beta}(x),
$$

where $\bar{g}_{\alpha \beta}(x)$ is an external (background) metric field and $h_{\alpha \beta}(x)$ is the variable of integration, also called quantum metric. Thus, the initial action is replaced by

$$
S_{0}(g) \rightarrow S_{0}(\bar{g}+h)
$$

The Faddeev-Popov $S_{F P}(\phi, \bar{g})$ action is constructed in the standard way [62]

$$
S_{F P}(\phi, \bar{g})=S_{0}(\bar{g}+h)+S_{g h}(\phi, \bar{g})+S_{g f}(\phi, \bar{g}),
$$

where $S_{g h}(\phi, \bar{g})$ is the ghost action and $S_{g f}(\phi, \bar{g})$ is the gauge-fixing action. In the presence of external metric $\bar{g}_{\alpha \beta}(x)$, they can be written as

$$
\begin{aligned}
S_{g h}(\phi, \bar{g})= & \int d x d y d z \sqrt{-\bar{g}(x)} \bar{C}^{\alpha}(x) H_{\alpha}^{\beta \gamma}(x, y ; \bar{g}, h) \\
& \times R_{\beta \gamma \sigma}(y, z ; \bar{g}+h) C^{\sigma}(z), \\
S_{g f}(\phi, \bar{g})= & \int d x \sqrt{-\bar{g}(x)} B^{\alpha}(x) \chi_{\alpha}(x ; \bar{g}, h),
\end{aligned}
$$

where

$$
H_{\alpha}^{\beta \gamma}(x, y ; \bar{g}, h)=\frac{\delta \chi_{\alpha}(x ; \bar{g}, h)}{\delta h_{\beta \gamma}(y)}
$$

and $\chi_{\alpha}(x ; \bar{g}, h)$ are the gauge-fixing functions, $\phi^{i}(x)=$ $\left\{h_{\alpha \beta}(x), B^{\alpha}(x), C^{\alpha}(x), \bar{C}^{\alpha}(x)\right\}$ is the set of quantum fields, $C^{\alpha}(x)$ and $\bar{C}^{\alpha}(x)$ are the ghost and antighost fields, respectively, and $B^{\alpha}(x)$ are the Nakanishi-Lautrup auxiliary fields. The Grassmann parity of all quantum fields are as follows:

$$
\varepsilon\left(h_{\alpha \beta}\right)=\varepsilon\left(B^{\alpha}\right)=0, \quad \varepsilon\left(\bar{C}^{\alpha}\right)=\varepsilon\left(C^{\alpha}\right)=1, \quad \varepsilon\left(\phi^{i}\right)=\varepsilon_{i} .
$$

The ghost numbers are

$$
\operatorname{gh}\left(B^{\alpha}\right)=\operatorname{gh}\left(h_{\alpha \beta}\right)=0, \quad \operatorname{gh}\left(C^{\alpha}\right)=1, \quad \operatorname{gh}\left(\bar{C}^{\alpha}\right)=-1 .
$$

Independent of gauge-fixing function choice, the action (10) is invariant under a global supersymmetry transformation, known as Becchi-Rouet-Stora-Tyutin symmetry $[63,64]$. The gravitational BRST transformations were introduced in $[4,65,66]$ and can be presented as

$$
\begin{aligned}
\delta_{B} h_{\alpha \beta}(x)= & -\left(C^{\sigma}(x) \partial_{\sigma} g_{\alpha \beta}(x)+g_{\alpha \sigma}(x) \partial_{\beta} C^{\sigma}(x)\right. \\
& \left.+g_{\sigma \beta}(x) \partial_{\alpha} C^{\sigma}(x)\right) \lambda, \\
\delta_{B} C^{\alpha}(x)= & C^{\sigma}(x) \partial_{\sigma} C^{\alpha}(x) \lambda, \\
\delta_{B} \bar{C}^{\alpha}(x)= & B^{\alpha}(x) \lambda, \\
\delta_{B} B^{\alpha}(x)= & 0,
\end{aligned}
$$

where $\lambda$ is a constant Grassmann parameter. In condensed notation, we can write the BRST transformations as

$$
\delta_{B} \phi^{i}(x)=R^{i}(x ; \phi, \bar{g}) \lambda, \quad \varepsilon\left(R^{i}\right)=\varepsilon_{i}+1,
$$

where $R^{i}=\left\{R_{\alpha \beta}^{(h)}, R_{(B)}^{\alpha}, R_{(C)}^{\alpha}, R_{(\bar{C})}^{\alpha}\right\}$ and

$$
\begin{aligned}
R_{\alpha \beta}^{(h)}(x ; \phi, \bar{g})= & -C^{\sigma}(x) \partial_{\sigma} g_{\alpha \beta}(x)-g_{\alpha \sigma}(x) \partial_{\beta} C^{\sigma}(x) \\
& -g_{\sigma \beta}(x) \partial_{\alpha} C^{\sigma}(x), \\
R_{(C)}^{\alpha}(x ; \phi, \bar{g})= & C^{\sigma}(x) \partial_{\sigma} C^{\alpha}(x), \\
R_{(\bar{C})}^{\alpha}(x ; \phi, \bar{g})= & B^{\alpha}(x) \\
R_{(B)}^{\alpha}(x ; \phi, \bar{g})= & 0 .
\end{aligned}
$$


The generating functional of Green functions is defined as

$$
\begin{aligned}
Z(J, \bar{g}) & =\int d \phi \exp \left\{\frac{i}{\hbar}\left[S_{F P}(\phi, \bar{g})+J \phi\right]\right\} \\
& =\exp \left\{\frac{i}{\hbar} W(J, \bar{g})\right\},
\end{aligned}
$$

where $W(J, \bar{g})$ is the generating functional of connected Green functions. In Eq. (17) the product of the sources $J_{i}(x)$ and the fields $\phi^{i}(x)$ was written in the condensed notation of DeWitt [61]. Explicitly,

$$
\begin{aligned}
J \phi & =\int d x J_{i}(x) \phi^{i}(x), \\
\text { where } J_{i}(x) & =\left\{J^{\mu \nu}, J_{\alpha}^{(B)}(x), \bar{J}_{\alpha}(x), J_{\alpha}(x)\right\}
\end{aligned}
$$

with the Grassmann parities $\varepsilon\left(J_{i}\right)=\varepsilon\left(\phi^{i}\right)$ and ghost numbers $\operatorname{gh}\left(J_{i}\right)=\operatorname{gh}\left(\phi^{i}\right)$.

The effective action $\Gamma(\Phi, \bar{g})$ is defined in terms of the Legendre transformation

$$
\Gamma(\Phi, \bar{g})=W(J, \bar{g})-J \Phi,
$$

where $\Phi=\left\{\Phi^{i}\right\}$ are the mean fields and the $J_{i}$ are the solution of the equation

$$
\frac{\delta W(J, \bar{g})}{\delta J_{i}}=\Phi^{i} .
$$

It is well known $[25,26]$ that the effective action is gauge independent on shell,

$$
\left.\delta \Gamma(\Phi, \bar{g})\right|_{\frac{\delta \Gamma(\Phi, \bar{g})}{\delta \Phi}=0}=0 .
$$

At this moment we have considered only the transformations of the quantum fields. However, the background metric also transforms together with the quantum fields in the so-called background field transformations. The rules of such transformation can be written, in the local formulation, as

$$
\begin{aligned}
\delta_{\omega} \bar{g}_{\alpha \beta}(x) & =-\partial_{\sigma} \bar{g}_{\alpha \beta}(x) \omega^{\sigma}-\bar{g}_{\alpha \sigma}(x) \partial_{\beta} \omega^{\sigma}-\bar{g}_{\sigma \beta}(x) \partial_{\alpha} \omega^{\sigma} \\
\delta_{\omega} h_{\alpha \beta}(x) & =-\partial_{\sigma} h_{\alpha \beta}(x) \omega^{\sigma}-h_{\alpha \sigma}(x) \partial_{\beta} \omega^{\sigma}-h_{\sigma \beta}(x) \partial_{\alpha} \omega^{\sigma} \\
\delta_{\omega} \bar{C}^{\alpha}(x) & =-\omega^{\sigma} \partial_{\sigma} \bar{C}^{\alpha}(x)+\bar{C}^{\sigma}(x) \partial_{\sigma} \omega^{\alpha} \\
\delta_{\omega} C^{\alpha}(x) & =-\omega^{\sigma} \partial_{\sigma} C^{\alpha}(x)+C^{\sigma}(x) \partial_{\sigma} \omega^{\alpha} \\
\delta_{\omega} B^{\alpha}(x) & =-\omega^{\sigma} \partial_{\sigma} B^{\alpha}(x)+B^{\sigma}(x) \partial_{\sigma} \omega^{\alpha}
\end{aligned}
$$

where $\omega^{\sigma}=\omega^{\sigma}(x)$ are arbitrary functions. The background field transformations have the same structure of tensor transformations for tensors of types $(0,2)$ and $(1,0)$. The background invariance of Faddeev-Popov action for quantum gravity is known [48] and reads

$$
\delta_{\omega} S_{F P}(\phi, \bar{g})=0 .
$$

A consequence of (23) is the gauge invariance of (17). Namely,

$$
\delta_{\omega} Z(J, \bar{g})=0 .
$$

From this expression it is possible to prove that $\Gamma(\Phi, \bar{g})$ is also gauge invariant

$$
\delta_{\omega} \Gamma(\Phi, \bar{g})=0 .
$$

In the next sections we will discuss this and other features for quantum gravity theories in the framework of the FRG approach.

\section{FRG APPROACH FOR QUANTUM GRAVITY THEORIES}

The main idea of a functional renormalization group is to use instead of $\Gamma$ an effective average action, $\Gamma_{k}$, where $k$ is a momentum-shell parameter [27], in a way that

$$
\lim _{k \rightarrow 0} \Gamma_{k}(\phi, \bar{g})=\Gamma(\phi, \bar{g}) .
$$

In order to obtain $\Gamma_{k}(\phi, \bar{g})$, we introduce the average action

$$
S_{k F P}(\phi, \bar{g})=S_{F P}(\phi, \bar{g})+S_{k}(\phi, \bar{g}),
$$

where $S_{k}(\phi, \bar{g})$ is the scale-dependent regulator action defined in curved spacetime

$$
S_{k}(\phi, \bar{g})=\int d x \sqrt{-\bar{g}(x)} \mathcal{L}_{k}(\phi, \bar{g})
$$

and the Lagrangian density is written as

$$
\begin{aligned}
\mathcal{L}_{k}(\phi, \bar{g})= & \frac{1}{2} h_{\alpha \beta}(x) R_{k}^{(1) \alpha \beta \gamma \delta}(x ; \bar{g}) h_{\gamma \delta}(x) \\
& +\bar{C}^{\alpha}(x) R_{k \alpha \beta}^{(2)}(x ; \bar{g}) C^{\beta}(x),
\end{aligned}
$$

where the regulator functions $R_{k}^{(1) \alpha \beta \gamma \delta}(x ; \bar{g})$ and $R_{k \alpha \beta}^{(2)}(x ; \bar{g})$ are dependent on the external fields $\bar{g}_{\alpha \beta}(x)$. The regulator functions obey the properties

$$
\lim _{k \rightarrow 0} R_{k}^{(1) \alpha \beta \gamma \delta}(x ; \bar{g})=0 \quad \text { and } \quad \lim _{k \rightarrow 0} R_{k \alpha \beta}^{(2)}(x ; \bar{g})=0,
$$

which means that the average action recovers the FaddeevPoppov action (10) in the limit when $k \rightarrow 0$. The regulator functions $R_{k}^{(1) \alpha \beta \gamma \delta}(x ; \bar{g})$ also obey, by construction, the symmetry properties 


$$
\begin{aligned}
R_{k}^{(1) \alpha \beta \gamma \delta}(x ; \bar{g}) & =R_{k}^{(1) \beta \alpha \gamma \delta}(x ; \bar{g})=R_{k}^{(1) \alpha \beta \delta \gamma}(x ; \bar{g}) \\
& =R_{k}^{(1) \gamma \delta \alpha \beta}(x ; \bar{g}) .
\end{aligned}
$$

We want to solve the problem of average action invariance under background field transformations, namely

$$
\delta_{\omega} S_{k F P}(\phi, \bar{g})=\delta_{\omega} S_{k}(\phi, \bar{g})=0,
$$

where the relation (23) is used.

In what follows, we present explicit calculation of variation of action (28). For this purpose, we write (32) as

$$
\begin{aligned}
\delta_{\omega} S_{k}(\phi, \bar{g})= & \int d x\left\{\delta_{\omega} \sqrt{-\bar{g}(x)} \mathcal{L}_{k}(\phi, \bar{g})\right. \\
& \left.+\sqrt{-\bar{g}(x)} \delta_{\omega} \mathcal{L}_{k}(\phi, \bar{g})\right\}=0 .
\end{aligned}
$$

For the first term in (33) we have

$$
\begin{aligned}
& \int d x \delta_{\omega} \sqrt{-\bar{g}(x)} \mathcal{L}_{k}(\phi, \bar{g}) \\
& =-\int d x \partial_{\sigma}\left(\sqrt{-\bar{g}(x)} \omega^{\sigma}\right) \mathcal{L}_{k}(\phi, \bar{g}) \\
& =\int d x \sqrt{-\bar{g}(x)} \omega^{\sigma} \partial_{\sigma} \mathcal{L}_{k}(\phi, \bar{g}),
\end{aligned}
$$

where integration by parts was used.

The variation of $\mathcal{L}_{k}(\phi, \bar{g})$ in the second term of Eq. (33) can be presented as

$$
\begin{aligned}
\delta_{\omega} \mathcal{L}_{k}(\phi, \bar{g})= & \frac{1}{2} \delta_{\omega} h_{\alpha \beta}(x) R_{k}^{(1) \alpha \beta \gamma \delta}(x ; \bar{g}) h_{\gamma \delta}(x)+\frac{1}{2} h_{\alpha \beta}(x) \delta_{\omega} R_{k}^{(1) \alpha \beta \gamma \delta}(x ; \bar{g}) h_{\gamma \delta}(x) \\
& +\frac{1}{2} h_{\alpha \beta}(x) R_{k}^{(1) \alpha \beta \gamma \delta}(x ; \bar{g}) \delta_{\omega} h_{\gamma \delta}(x)+\delta_{\omega} \bar{C}^{\alpha}(x) R_{k \alpha \beta}^{(2)}(x ; \bar{g}) C^{\beta}(x) \\
& +\bar{C}^{\alpha}(x) \delta_{\omega} R_{k \alpha \beta}^{(2)}(x ; \bar{g}) C^{\beta}(x)+\bar{C}^{\alpha}(x) R_{k \alpha \beta}^{(2)}(x ; \bar{g}) \delta_{\omega} C^{\beta}(x) .
\end{aligned}
$$

In terms of transformations (22), the above expression reads

$$
\begin{aligned}
\delta_{\omega} \mathcal{L}_{k}(\phi, \bar{g})= & -\frac{1}{2}\left(\partial_{\sigma} h_{\alpha \beta}(x) \omega^{\sigma}+h_{\alpha \sigma}(x) \partial_{\beta} \omega^{\sigma}+h_{\sigma \beta}(x) \partial_{\alpha} \omega^{\sigma}\right) R_{k}^{(1) \alpha \beta \gamma \delta}(x ; \bar{g}) h_{\gamma \delta}(x) \\
& -\frac{1}{2} h_{\alpha \beta} R_{k}^{(1) \alpha \beta \gamma \delta}(x ; \bar{g})\left(\partial_{\sigma} h_{\gamma \delta}(x) \omega^{\sigma}+h_{\gamma \sigma}(x) \partial_{\delta} \omega^{\sigma}+h_{\sigma \delta}(x) \partial_{\gamma} \omega^{\sigma}\right) \\
& +\frac{1}{2} h_{\alpha \beta}(x) \delta_{\omega} R_{k}^{(1) \alpha \beta \gamma \delta}(x ; \bar{g}) h_{\gamma \delta}(x)+\bar{C}^{\alpha}(x) \delta_{\omega} R_{k \alpha \beta}^{(2)}(x ; \bar{g}) C^{\beta}(x) \\
& +\left(-\omega^{\sigma} \partial_{\sigma} \bar{C}^{\alpha}(x)+\bar{C}^{\sigma}(x) \partial_{\sigma} \omega^{\alpha}\right) R_{k \alpha \beta}^{(2)}(x ; \bar{g}) C^{\beta}(x) \\
& +\bar{C}^{\alpha}(x) R_{k \alpha \beta}^{(2)}(x ; \bar{g})\left(-\omega^{\sigma} \partial_{\sigma} C^{\beta}(x)+C^{\sigma}(x) \partial_{\sigma} \omega^{\beta}\right) .
\end{aligned}
$$

Thus, by means of Eqs. (34) and (36) the variation of the action can be written in the compact way

$$
\delta_{\omega} S_{k}(\phi, \bar{g})=\int d x \sqrt{-\bar{g}(x)}\left\{\frac{1}{2} h_{\alpha \beta}(x) M_{\omega k}^{(1) \alpha \beta \gamma \delta}(x ; \bar{g}) h_{\gamma \delta}(x)+\bar{C}^{\alpha}(x) M_{\omega k \alpha \beta}^{(2)}(x ; \bar{g}) C^{\beta}(x)\right\},
$$

where

$$
\begin{aligned}
M_{\omega k}^{(1) \alpha \beta \gamma \delta}(x ; \bar{g})= & \delta_{\omega} R_{k}^{(1) \alpha \beta \gamma \delta}(x ; \bar{g})+\omega^{\sigma} \partial_{\sigma} R_{k}^{(1) \alpha \beta \gamma \delta}(x ; \bar{g})-\partial_{\sigma} \omega^{\alpha} R_{k}^{(1) \sigma \beta \gamma \delta}(x ; \bar{g}) \\
& -\partial_{\sigma} \omega^{\beta} R_{k}^{(1) \alpha \sigma \gamma \delta}(x ; \bar{g})-\partial_{\sigma} \omega^{\gamma} R_{k}^{(1) \alpha \beta \sigma \delta}(x ; \bar{g})-\partial_{\sigma} \omega^{\delta} R_{k}^{(1) \alpha \beta \gamma \sigma}(x ; \bar{g})
\end{aligned}
$$

and

$$
M_{\omega k \alpha \beta}^{(2)}(x ; \bar{g})=\delta_{\omega} R_{k \alpha \beta}^{(2)}(x ; \bar{g})+\omega^{\sigma} \partial_{\sigma} R_{k \alpha \beta}^{(2)}(x ; \bar{g})+R_{k \sigma \beta}^{(2)}(x ; \bar{g}) \partial_{\alpha} \omega^{\sigma}+R_{k \alpha \sigma}^{(2)}(x ; \bar{g}) \partial_{\beta} \omega^{\sigma} .
$$


In order to ensure the invariance of (28), it is necessary that the following conditions are satisfied:

$$
M_{\omega k}^{(1) \alpha \beta \gamma \delta}(x ; \bar{g})=0 \quad \text { and } \quad M_{\omega k \alpha \beta}^{(2)}(x ; \bar{g})=0 .
$$

As a result, we obtain expressions for the variation of the regulator functions,

$$
\begin{aligned}
\delta_{\omega} R_{k}^{(1) \alpha \beta \gamma \delta}(x ; \bar{g})= & -\omega^{\sigma} \partial_{\sigma} R_{k}^{(1) \alpha \beta \gamma \delta}(x ; \bar{g}) \\
& +R_{k}^{(1) \sigma \beta \gamma \delta}(x ; \bar{g}) \partial_{\sigma} \omega^{\alpha}+R_{k}^{(1) \alpha \sigma \gamma \delta}(x ; \bar{g}) \partial_{\sigma} \omega^{\beta} \\
& +R_{k}^{(1) \alpha \beta \sigma \delta}(x ; \bar{g}) \partial_{\sigma} \omega^{\gamma}+R_{k}^{(1) \alpha \beta \gamma \sigma}(x ; \bar{g}) \partial_{\sigma} \omega^{\delta}
\end{aligned}
$$

and

$$
\begin{aligned}
\delta_{\omega} R_{k \alpha \beta}^{(2)}(x ; \bar{g})= & -\omega^{\sigma} \partial_{\sigma} R_{k \alpha \beta}^{(2)}(x ; \bar{g})-R_{k \sigma \beta}^{(2)}(x ; \bar{g}) \partial_{\alpha} \omega^{\sigma} \\
& -R_{k \alpha \sigma}^{(2)}(x ; \bar{g}) \partial_{\beta} \omega^{\sigma} .
\end{aligned}
$$

If the relations (40) and (41) are fulfilled, then the action $S_{k}(\phi, \bar{g})$ is invariant under background field transformations. Therefore, the regulator functions should have a tensor structure in order to ensure the invariance. Thus, taking into account the symmetry properties presented in (31) we can propose the following solutions for the regulator functions:

$$
\begin{aligned}
R_{k}^{(1) \alpha \beta \gamma \delta}(x ; \bar{g})= & \bar{g}^{\alpha \beta}(x) \bar{g}^{\gamma \delta}(x) R_{k}^{(1)}(\bar{\square}) \\
& +\left(\bar{g}^{\alpha \gamma}(x) \bar{g}^{\beta \delta}(x)+\bar{g}^{\alpha \delta}(x) \bar{g}^{\beta \gamma}(x)\right) Q_{k}(\bar{\square})
\end{aligned}
$$

and

$$
R_{k \alpha \beta}^{(2)}(x ; \bar{g})=\bar{g}_{\alpha \beta} R_{k}^{(2)}(\bar{\square}),
$$

where $R_{k}^{(1,2)}(\bar{\square})$ and $Q_{k}(\bar{\square})$ are scalar functions and $\bar{\square}$ is the d'Alembertian operator defined in terms of the covariant derivatives of the background metric:

$$
\bar{\square}=\bar{g}^{\mu \nu} \bar{\nabla}_{\mu} \bar{\nabla}_{\nu},
$$

with the metricity property

$$
\bar{\nabla}_{\tau} \bar{g}_{\mu \nu}=0
$$

It is possible to show that (42) and (43) present the same variational structure of (40) and (41), respectively. By using the inverse background metric variation

$$
\delta_{\omega} \bar{g}^{\mu \nu}(x)=-\omega^{\sigma} \partial_{\sigma} \bar{g}^{\mu \nu}(x)+\bar{g}^{\mu \sigma}(x) \partial_{\sigma} \omega^{\nu}+\bar{g}^{\sigma \nu}(x) \partial_{\sigma} \omega^{\mu},
$$

we have

$$
\begin{aligned}
\delta_{\omega} R_{k}^{(1) \alpha \beta \gamma \delta}(x ; \bar{g})= & \left(-\omega^{\sigma} \partial_{\sigma} \bar{g}^{\alpha \beta}(x)+\bar{g}^{\alpha \sigma}(x) \partial_{\sigma} \omega^{\beta}+\bar{g}^{\sigma \beta}(x) \partial_{\sigma} \omega^{\alpha}\right) \bar{g}^{\gamma \delta}(x) R_{k}^{(1)}(\bar{\square}) \\
& +\bar{g}^{\alpha \beta}(x)\left(-\omega^{\sigma} \partial_{\sigma} \bar{g}^{\gamma \delta}(x)+\bar{g}^{\gamma \sigma}(x) \partial_{\sigma} \omega^{\delta}+\bar{g}^{\sigma \delta}(x) \partial_{\sigma} \omega^{\gamma}\right) R_{k}^{(1)}(\bar{\square}) \\
& +\left(-\omega^{\sigma} \partial_{\sigma} \bar{g}^{\alpha \gamma}(x)+\bar{g}^{\alpha \sigma}(x) \partial_{\sigma} \omega^{\gamma}+\bar{g}^{\sigma \gamma}(x) \partial_{\sigma} \omega^{\alpha}\right) \bar{g}^{\beta \delta}(x) Q_{k}(\bar{\square}) \\
& +\bar{g}^{\alpha \gamma}(x)\left(-\omega^{\sigma} \partial_{\sigma} \bar{g}^{\beta \delta}(x)+\bar{g}^{\beta \sigma}(x) \partial_{\sigma} \omega^{\delta}+\bar{g}^{\sigma \delta}(x) \partial_{\sigma} \omega^{\beta}\right) Q_{k}(\bar{\square}) \\
& +\left(-\omega^{\sigma} \partial_{\sigma} \bar{g}^{\alpha \delta}(x)+\bar{g}^{\alpha \sigma}(x) \partial_{\sigma} \omega^{\delta}+\bar{g}^{\sigma \delta}(x) \partial_{\sigma} \omega^{\alpha}\right) \bar{g}^{\beta \gamma}(x) Q_{k}(\bar{\square}) \\
& +\bar{g}^{\alpha \delta}(x)\left(-\omega^{\sigma} \partial_{\sigma} \bar{g}^{\beta \gamma}(x)+\bar{g}^{\beta \sigma}(x) \partial_{\sigma} \omega^{\gamma}+\bar{g}^{\sigma \gamma}(x) \delta_{\sigma} \omega^{\beta}\right) Q_{k}(\bar{\square}) \\
& -\bar{g}^{\alpha \beta}(x) \bar{g}^{\gamma \delta}(x) \omega^{\sigma} \partial_{\sigma} R_{k}^{(1)}(\bar{\square})-\bar{g}^{\alpha \gamma}(x) \bar{g}^{\beta \delta}(x) \omega^{\sigma} \partial_{\sigma} Q_{k}(\bar{\square}) \\
& -\bar{g}^{\alpha \delta}(x) \bar{g}^{\beta \gamma}(x) \omega^{\sigma} \partial_{\sigma} Q_{k}(\bar{\square}) .
\end{aligned}
$$

The derivatives in metric tensor and in functions $R_{k}^{(1)}(\bar{\square})$ and $Q_{k}(\bar{\square})$ can be combined to obtain

$$
\begin{aligned}
\delta_{\omega} R_{k}^{(1) \alpha \beta \gamma \delta}(x ; \bar{g})= & \left(\bar{g}^{\alpha \sigma}(x) \partial_{\sigma} \omega^{\beta}+\bar{g}^{\sigma \beta}(x) \partial_{\sigma} \omega^{\alpha}\right) \bar{g}^{\gamma \delta}(x) R_{k}^{(1)}(\bar{\square})+\bar{g}^{\alpha \beta}(x)\left(\bar{g}^{\gamma \sigma}(x) \partial_{\sigma} \omega^{\delta}+\bar{g}^{\sigma \delta}(x) \partial_{\sigma} \omega^{\gamma}\right) R_{k}^{(1)}(\bar{\square}) \\
& +\left(\bar{g}^{\alpha \sigma}(x) \partial_{\sigma} \omega^{\gamma}+\bar{g}^{\sigma \gamma}(x) \partial_{\sigma} \omega^{\alpha}\right) \bar{g}^{\beta \delta}(x) Q_{k}(\bar{\square})+\bar{g}^{\alpha \gamma}(x)\left(\bar{g}^{\beta \sigma}(x) \partial_{\sigma} \omega^{\delta}+\bar{g}^{\sigma \delta}(x) \partial_{\sigma} \omega^{\beta}\right) Q_{k}(\bar{\square}) \\
& +\left(\bar{g}^{\alpha \sigma}(x) \partial_{\sigma} \omega^{\delta}+\bar{g}^{\sigma \delta}(x) \partial_{\sigma} \omega^{\alpha}\right) \bar{g}^{\beta \gamma}(x) Q_{k}(\bar{\square})+\bar{g}^{\alpha \delta}(x)\left(+\bar{g}^{\beta \sigma}(x) \partial_{\sigma} \omega^{\delta}+\bar{g}^{\sigma \gamma}(x) \delta_{\sigma} \omega^{\beta}\right) Q_{k}(\bar{\square}) \\
& -\omega^{\sigma} \partial_{\sigma}\left(\bar{g}^{\alpha \beta}(x) \bar{g}^{\gamma \delta}(x) R_{k}^{(1)}(\bar{\square})\right)-\omega^{\sigma} \partial_{\sigma}\left(\bar{g}^{\alpha \gamma}(x) \bar{g}^{\beta \delta}(x) Q_{k}(\bar{\square})\right)-\omega^{\sigma} \partial_{\sigma}\left(\bar{g}^{\alpha \delta}(x) \bar{g}^{\beta \gamma}(x) Q_{k}(\bar{\square})\right) .
\end{aligned}
$$

As a result, it is possible to see that (40) is satisfied. 
For the second function its variation can be expressed, after some algebra, as

$$
\begin{aligned}
\delta_{\omega} R_{k \alpha \beta}^{(2)}(x ; \bar{g})= & -\omega^{\sigma} \partial_{\sigma} \bar{g}_{\alpha \beta} R_{k}^{(2)}(\bar{\square})-\partial_{\alpha} \omega^{\sigma} \bar{g}_{\sigma \beta}(x) R_{k}^{(2)}(\bar{\square}) \\
& -\partial_{\beta} \omega^{\sigma} \bar{g}_{\alpha \sigma}(x) R_{k}^{(2)}(\bar{\square}) \\
& -\omega^{\sigma} \bar{g}_{\alpha \beta}(x) \partial_{\sigma} R_{k}^{(2)}(\bar{\square}) .
\end{aligned}
$$

The combination of derivatives in the metric tensor and in the scalar function leads to

$$
\begin{aligned}
\delta_{\omega} R_{k \alpha \beta}^{(2)}(x ; \bar{g})= & -\omega^{\sigma} \partial_{\sigma}\left(\bar{g}_{\alpha \beta}(x) R_{k}^{(2)}(\bar{\square})\right) \\
& -\partial_{\alpha} \omega^{\sigma} \bar{g}_{\sigma \beta}(x) R_{k}^{(2)}(\bar{\square}) \\
& -\partial_{\beta} \omega^{\sigma} \bar{g}_{\alpha \sigma}(x) R_{k}^{(2)}(\bar{\square}),
\end{aligned}
$$

which has the same structure as (41).

Finally, the scale-dependent regulator Lagrangian density (29) in terms of (42) and (43) reads

$$
\begin{aligned}
\mathcal{L}_{k}(\phi, \bar{g})= & \frac{1}{2} h_{\alpha \beta}(x)\left[\bar{g}^{\alpha \beta}(x) \bar{g}^{\gamma \delta}(x) R_{k}^{(1)}(\bar{\square})\right. \\
& \left.+\left(\bar{g}^{\alpha \gamma}(x) \bar{g}^{\beta \delta}(x)+\bar{g}^{\alpha \delta}(x) \bar{g}^{\beta \gamma}(x)\right) Q_{k}(\bar{\square})\right] h_{\gamma \delta}(x) \\
& +\bar{C}^{\alpha}(x) \bar{g}_{\alpha \beta}(x) R_{k}^{(2)}(\bar{\square}) C^{\beta}(x),
\end{aligned}
$$

which maintains the background field symmetry, $\delta_{\omega} S_{k}(\phi, \bar{g})=0$.

\section{GAUGE DEPENDENCE OF EFFECTIVE AVERAGE ACTION}

In order to understand the gauge invariance and gauge dependence problems in the background field method, we shall consider the generating functionals of Green functions

$$
\begin{aligned}
Z_{k \Psi}(J, \bar{g})= & \int d \phi \exp \left\{\frac { i } { \hbar } \left[S_{0}(h+\bar{g})+\hat{R}(\phi, \bar{g}) \Psi(\phi, \bar{g})\right.\right. \\
& \left.\left.+S_{k}(\phi, \bar{g})+J \phi\right]\right\} \\
= & \int d \phi \exp \left\{\frac{i}{\hbar}\left[S_{k F P}(\phi, \bar{g})+J \phi\right]\right\} \\
= & \exp \left\{\frac{i}{\hbar} W_{k \Psi}(J, \bar{g})\right\},
\end{aligned}
$$

where

$$
\Psi(\phi, \bar{g})=\int d x \sqrt{-\bar{g}(x)} \bar{C}^{\alpha}(x) \chi_{\alpha}(x ; h, \bar{g})
$$

is the fermionic gauge-fixing functional and

$$
\hat{R}(\phi, \bar{g})=\int d x \frac{\delta_{r}}{\delta \phi^{i}(x)} R^{i}(x ; \phi, \bar{g})
$$

is the generator of BRST transformations (15).

As far as we saw in the previous section, the regulator action (28) does not depend on the gauge $\Psi(\phi, \bar{g})$. Now, we shall consider another choice of gauge-fixing functional $\Psi \rightarrow \Psi+\delta \Psi$ and set $J=0$ in (52). Thus,

$$
\begin{aligned}
Z_{k \Psi+\delta \Psi}(\bar{g}) & =\int d \phi \exp \left\{\frac{i}{\hbar}\left[S_{k F P}(\phi, \bar{g})+\hat{R}(\phi, \bar{g}) \delta \Psi(\phi, \bar{g})\right]\right\} \\
& =\exp \left\{\frac{i}{\hbar} W_{k \Psi+\delta \Psi}(\bar{g})\right\}
\end{aligned}
$$

where

$$
\delta \Psi=\delta \Psi(\phi, \bar{g})=\int d x \sqrt{-\bar{g}(x)} \bar{C}^{\alpha}(x) \delta \chi_{\alpha}(h, \bar{g}) .
$$

We will try to compensate the additional term $\hat{R} \delta \Psi$ in (55). To do this, we change the variables in the functional integral related to the symmetries of action $S_{F P}(\phi, \bar{g})$, namely the BRST symmetry and the background gauge invariance. First, we shall consider the BRST symmetry (14), but trading the constant parameter $\lambda$ by a functional $\Lambda=\Lambda(\phi, \bar{g})$. The variation of (28) under such transformation is the following:

$$
\delta_{B} S_{k}(\phi, \bar{g})=\int d^{4} x \sqrt{-\bar{g}(x)}\left\{\delta_{B} \mathcal{L}_{k}(\phi, \bar{g})\right\},
$$

where

$$
\begin{aligned}
\delta_{B} \mathcal{L}_{k}= & \frac{1}{2} \delta_{B} h_{\alpha \beta}(x) R_{k}^{(1) \alpha \beta \gamma \delta}(x ; \bar{g}) h_{\gamma \delta}(x) \\
& +\frac{1}{2} h_{\alpha \beta}(x) R_{k}^{(1) \alpha \beta \gamma \delta}(x ; \bar{g}) \delta_{B} h_{\gamma \delta}(x) \\
& +\delta_{B} \bar{C}^{\alpha}(x) R_{k \alpha \beta}^{(2)}(x ; \bar{g}) C^{\beta}(x)+\bar{C}^{\alpha}(x) R_{k \alpha \beta}^{(2)}(x ; \bar{g}) \delta_{B} C^{\beta}(x) .
\end{aligned}
$$

After some algebra, $\delta_{B} \mathcal{L}_{k}(\phi, \bar{g})$ reads as

$$
\begin{aligned}
\delta_{B} \mathcal{L}_{k}= & -\frac{1}{2}\left(C^{\sigma}(x) \partial_{\sigma} g_{\alpha \beta}(x)+g_{\alpha \sigma}(x) \partial_{\beta} C^{\sigma}(x)+g_{\sigma \beta}(x) \partial_{\alpha} C^{\sigma}(x)\right) \Lambda R_{k}^{(1) \alpha \beta \gamma \delta}(x ; \bar{g}) h_{\gamma \delta}(x) \\
& -\frac{1}{2} h_{\alpha \beta}(x) R_{k}^{(1) \alpha \beta \gamma \delta}(x ; \bar{g})\left(C^{\sigma}(x) \partial_{\sigma} g_{\gamma \delta}(x)+g_{\gamma \sigma}(x) \partial_{\delta} C^{\sigma}(x)+g_{\sigma \delta}(x) \partial_{\gamma} C^{\sigma}(x)\right) \Lambda \\
& +B^{\alpha}(x) \Lambda R_{k \alpha \beta}^{(2)}(x ; \bar{g}) C^{\beta}(x)+\bar{C}^{\alpha}(x) R_{k \alpha \beta}^{(2)}(x ; \bar{g}) C^{\sigma}(x) \partial_{\sigma} C^{\beta}(x) \Lambda .
\end{aligned}
$$


From the above expression, it is clear that the action $S_{k}(\phi, \bar{g})$ is not invariant under BRST transformations $\delta_{B} S_{k}(\phi, \bar{g}) \neq 0$. The Jacobian $J_{1}$ of such transformation can be obtained in the standard way

$$
J_{1}=\exp \left\{\int d x\left[\frac{\delta\left(\delta_{B} h_{\alpha \beta}(x)\right)}{\delta h_{\alpha \beta}(x)}-\frac{\delta\left(\delta_{B} C^{\alpha}(x)\right)}{\delta C^{\alpha}(x)}-\frac{\delta\left(\delta_{B} \bar{C}^{\alpha}(x)\right)}{\delta \bar{C}^{\alpha}(x)}\right]\right\},
$$

where the functional derivatives are

$$
\begin{aligned}
\frac{\delta\left(\delta_{B} h_{\alpha \beta}(x)\right)}{\delta h_{\alpha \beta}(x)}= & -\frac{D(D+1)}{2} \delta(0) C^{\sigma}(x) \partial_{\sigma} \Lambda(\phi, \bar{g})-\frac{(D+1)(D-2)}{2} \delta(0) \partial_{\sigma} C^{\sigma}(x) \Lambda(\phi, \bar{g}) \\
& -\left[C(x)^{\sigma} \partial_{\sigma} g_{\alpha \beta}(x)+g_{\alpha \sigma}(x) \partial_{\beta} C^{\sigma}(x)+g_{\sigma \beta}(x) \partial_{\alpha} C^{\sigma}(x)\right] \frac{\delta \Lambda(\phi, \bar{g})}{\delta h_{\alpha \beta}(x)}, \\
\frac{\delta\left(\delta_{B} C^{\alpha}(x)\right)}{\delta C^{\alpha}(x)}= & (D+1) \delta(0) \partial_{\sigma} C^{\sigma}(x) \Lambda(\phi, \bar{g})+D \delta(0) C^{\sigma}(x) \partial_{\sigma} \Lambda(\phi, \bar{g})+C^{\sigma}(x) \partial_{\sigma} C^{\alpha}(x) \frac{\delta \Lambda(\phi, \bar{g})}{\delta C^{\alpha}(x)}, \\
\frac{\delta\left(\delta_{B} \bar{C}^{\alpha}(x)\right)}{\delta \bar{C}^{\alpha}(x)}= & B^{\alpha}(x) \frac{\delta \Lambda(\phi, \bar{g})}{\delta \bar{C}^{\alpha}(x)} .
\end{aligned}
$$

It is possible to choose a regularization scheme such that $\delta(0)=0$. As a result, the Jacobian for BRST transformations is

$$
J_{1}=\exp \left\{\int d x\left[R_{\alpha \beta}^{(h)}(x ; \phi, \bar{g}) \frac{\delta \Lambda(\phi, \bar{g})}{\delta h_{\alpha \beta}(x)}-R_{(C)}^{\alpha}(x ; \phi, \bar{g}) \frac{\delta \Lambda(\phi, \bar{g})}{\delta C^{\alpha}(x)}-R_{(\bar{C})}^{\alpha}(x ; \phi, \bar{g}) \frac{\delta \Lambda(\phi, \bar{g})}{\delta \bar{C}^{\alpha}(x)}\right]\right\},
$$

where (15) is used.

It is also interesting to consider the background gauge transformation related to expressions (22). As far as the regulator functions transform as (40) and (41), the action $S_{k}(\phi, \bar{g})$ is invariant under such transformation. But now, instead of functions $\omega=\omega^{\sigma}(x)$ we shall consider the functional $\Omega^{\sigma}=\Omega^{\sigma}(x, \phi, \bar{g})$. The action (28) remains invariant, and the corresponding
Jacobian of this transformation can be obtained as before

$$
J_{2}=\exp \left\{\int d x \frac{\delta\left(\delta_{\Omega} h_{\alpha \beta}(x)\right)}{\delta h_{\alpha \beta}(x)}-\frac{\delta\left(\delta_{\Omega} C^{\alpha}(x)\right)}{\delta C^{\alpha}(x)}-\frac{\delta\left(\delta_{\Omega} \bar{C}^{\alpha}(x)\right)}{\delta \bar{C}^{\alpha}(x)}\right\},
$$

with the following functional derivatives:

$$
\begin{aligned}
& \frac{\delta\left(\delta_{\Omega} h_{\alpha \beta}(x)\right)}{\delta h_{\alpha \beta}(x)}=\frac{(D+1)(D-2)}{2} \delta(0) \partial_{\sigma} \Omega^{\sigma}(x, \phi, \bar{g})-\left(\partial_{\sigma} h_{\alpha \beta}(x)+h_{\alpha \sigma}(x) \partial_{\beta}+h_{\sigma \beta}(x) \partial_{\alpha}\right) \frac{\delta \Omega^{\sigma}(x, \phi, \bar{g})}{\delta h_{\alpha \beta}(x)}, \\
& \frac{\delta\left(\delta_{\Omega} \bar{C}^{\alpha}(x)\right)}{\delta \bar{C}^{\alpha}(x)}=(D+1) \delta(0) \partial_{\sigma} \Omega^{\sigma}(x, \phi, \bar{g})-\frac{\delta \Omega^{\sigma}(x, \phi, \bar{g})}{\delta \bar{C}^{\alpha}(x)} \partial_{\sigma} \bar{C}^{\alpha}(x)-\bar{C}^{\sigma}(x) \partial_{\sigma} \frac{\delta \Omega^{\alpha}(x, \phi, \bar{g})}{\delta \bar{C}^{\alpha}(x)}, \\
& \frac{\delta\left(\delta_{\Omega} C^{\alpha}(x)\right)}{\delta C^{\alpha}(x)}=(D+1) \delta(0) \partial_{\sigma} \Omega^{\sigma}(x, \phi, \bar{g})-\frac{\delta \Omega^{\sigma}(x, \phi, \bar{g})}{\delta C^{\alpha}(x)} \partial_{\sigma} C^{\alpha}(x)-C^{\sigma}(x) \partial_{\sigma} \frac{\delta \Omega^{\alpha}(x, \phi, \bar{g})}{\delta C^{\alpha}(x)} .
\end{aligned}
$$

As before, $\delta(0)=0$. Thus, the Jacobian for background gauge transformations reads

$$
\begin{aligned}
J_{2}= & \exp \left\{\int d x \left[-\left(\partial_{\sigma} h_{\alpha \beta}(x)+h_{\alpha \sigma}(x) \partial_{\beta}+h_{\sigma \beta}(x) \partial_{\alpha}\right) \frac{\delta \Omega^{\sigma}(x, \phi, \bar{g})}{\delta h_{\alpha \beta}(x)}+\frac{\delta \Omega^{\sigma}(x, \phi, \bar{g})}{\delta \bar{C}^{\alpha}(x)} \partial_{\sigma} \bar{C}^{\alpha}(x)+\bar{C}^{\sigma}(x) \partial_{\sigma} \frac{\delta \Omega^{\alpha}(x, \phi, \bar{g})}{\delta \bar{C}^{\alpha}(x)}\right.\right. \\
& \left.\left.+\frac{\delta \Omega^{\sigma}(x, \phi, \bar{g})}{\delta C^{\alpha}(x)} \partial_{\sigma} C^{\alpha}(x)+C^{\sigma}(x) \partial_{\sigma} \frac{\delta \Omega^{\alpha}(x, \phi, \bar{g})}{\delta C^{\alpha}(x)}\right]\right\}
\end{aligned}
$$


If it is possible to fulfill the condition

$$
J_{1} J_{2} \exp \left\{\frac{i}{\hbar} \int d x\left[\hat{R}(\phi, \bar{g}) \delta \Psi(\phi, \bar{g})+\delta_{B} S_{k}(\phi, \bar{g})\right]\right\}=1
$$

the generating vacuum functional $Z_{k \Psi}(\bar{g})$ does not depend on the gauge fixing functional $\Psi$. In order to verify that, let us expand the functionals $\Lambda$ and $\Omega$, with Grassmann parity $\varepsilon(\Lambda)=1$ and $\varepsilon(\Omega)=0$ and ghost numbers $\operatorname{gh}(\Lambda)=-1$ and $\operatorname{gh}(\Omega)=0$ in the lower power of ghost fields

$$
\Lambda=\Lambda^{(1)}+\Lambda^{(3)}, \quad \Omega^{\sigma}=\Omega^{\sigma(0)}+\Omega^{\sigma(2)},
$$

where

$$
\begin{aligned}
\Lambda^{(1)} & =\int d x \bar{C}^{\alpha}(x) \lambda_{\alpha}^{(1)}(x, h, \bar{g}), \\
\Lambda^{(3)} & =\int d x \frac{1}{2} \bar{C}^{\alpha}(x) \bar{C}^{\beta}(x) \lambda_{\alpha \beta \gamma}^{(3)}(x, h, \bar{g}) C^{\gamma}(x), \\
\Omega^{\sigma(0)}(x) & =\Omega^{\sigma(0)}(x, h, \bar{g}), \\
\Omega^{\sigma(2)}(x, h, \bar{g}) & =\bar{C}^{\alpha}(x) \omega_{\alpha \beta}^{\sigma(2)}(x, h, \bar{g}) C^{\beta}(x) .
\end{aligned}
$$

The terms that vanish in (68) and do not depend on ghost fields lead to

$$
\left(\partial_{\sigma} h_{\alpha \beta}(x)+h_{\alpha \sigma}(x) \partial_{\beta}+h_{\sigma \beta}(x) \partial_{\alpha}\right) \frac{\delta \Omega^{\sigma(0)}(x, h, \bar{g})}{\delta h_{\alpha \beta}(x)}=0 .
$$

Analyzing the terms which are linear in the antighost fields, which contains the auxiliary fields $B(x)$, we obtain

$$
\lambda_{\alpha}^{(1)}(x, h, \bar{g})=\frac{i}{\hbar} \delta \chi_{\alpha}(x, h, \bar{g})
$$

and

$$
\begin{aligned}
\lambda_{\alpha \beta \gamma}^{(3)}(x, h, \bar{g})= & \lambda_{\alpha}^{(1)}(h, \bar{g}) R_{k \beta \gamma}^{(2)}(x ; \bar{g}) \\
& -\lambda_{\beta}^{(1)}(h, \bar{g}) R_{k \alpha \gamma}^{(2)}(x ; \bar{g}),
\end{aligned}
$$

where

$$
\lambda_{\alpha}^{(1)}(h, \bar{g})=\int d x \lambda_{\alpha}^{(1)}(x, h, \bar{g})
$$

Now, the vanishing terms with structure $\bar{C}(x) C(x)$ can be related to the second order of $\Omega^{\sigma}(x, h, \bar{g})$ functional leading to a differential equation for $\omega_{\alpha \beta}^{\sigma(2)}(x, h, \bar{g})$,

$$
\begin{aligned}
\partial_{\sigma} \omega_{\alpha \beta}^{\sigma(2)}(x, h, \bar{g})= & \frac{i}{2 \hbar}\left[\partial_{\beta} g_{\tau \sigma}(x) \lambda_{\alpha}^{(1)}(h, \bar{g}) R^{(1) \tau \sigma \gamma \delta}(x ; \bar{g}) h_{\gamma \delta}(x)\right. \\
& \left.+h_{\tau \sigma}(x) R^{(1) \tau \sigma \gamma \delta}(x ; \bar{g}) \partial_{\beta} g_{\gamma \delta}(x) \lambda_{\alpha}^{(1)}(h, \bar{g})\right] .
\end{aligned}
$$

From Eq. (74) since $\Omega^{\sigma(0)}(x, h, \bar{g})$ is an arbitrary function we can not have just one particular solution. In addition, the $\lambda_{\alpha \beta \gamma}^{(3)}$ relation in (76) creates a nonlocal term of structure $B \bar{C} \bar{C} C C$ which can be only eliminated if we consider a new functional $\Lambda$ of higher orders in ghost fields. Even so, this process would repeat endlessly. The only case left for us is to consider the simple solution when $\Omega^{\sigma}=0$ and $\Lambda=\Lambda^{(1)}$; we have the result

$$
\begin{aligned}
Z_{k \Psi+\delta \Psi}(\bar{g}) & =\int d \phi \exp \left\{\frac{i}{\hbar}\left[S_{k F P}(\phi, \bar{g})+\delta S_{k F P}(\phi)\right]\right\}, \\
Z_{k \Psi}(\bar{g}) & \neq Z_{k \Psi+\delta \Psi}(\bar{g}) .
\end{aligned}
$$

As a final result, the vacuum functional in the FRG approach for gravity theories depends on the gauge fixing even on shell, which leads to a gauge-dependent $S$-matrix.

\section{CONCLUSIONS AND PERSPECTIVES}

We explored the problem of gauge invariance and the gauge-fixing dependence using the background field formalism, for gravity theories in the FRG framework. It was shown that the background field invariance is achieved when the regulator functions are chosen to have the tensor structure. Nevertheless, even in this case the on-shell gauge dependence cannot be cured in the standard FRG approach which we dealt with. In this respect the situation is qualitatively the same as in the Yang-Mills theories, as was discussed in [15].

The on-shell gauge dependence takes place due to the fact that the regulator action (28) is not BRST invariant. It turns out that this is a fundamentally important feature, that can be changed only by trading the standard and conventional FRG framework to an alternative one, which is based on the use of composite operators for constructing the regulator action. Unfortunately, until now there is no way to perform practical calculations in this alternative formulation. For this reason, taking our present results into account, it remains unclear whether the quantum gravity results obtained within the FRG formalism can have a reasonable physical interpretation. One can expect that all predictions of this formalism will depend on an arbitrary choice of the gauge fixing. Thus, one can, in principle, provide any desirable result, but the value of this output is not clear. Alternatively, there should be found some physical reason to claim that one special gauge fixing is "correct" or "preferred" for some reason, but at the moment it is unclear what this reason could be, since the original theory is gauge (diffeomorphism) invariant. 
It is worthwhile to comment on the effect of the on-shell gauge-fixing dependence on the nonuniversality of the renormalization group flow in the framework of the functional renormalization group approach. First of all, the complete understanding of this issue requires a detailed analysis and, in principle, explicit calculations in the sufficiently general model of quantum gravity. This calculation is a separate problem that is beyond the scope of the present work. At the same time, we can give some strong arguments in favor of such a gauge-fixing dependence for both renormalization group flow and the fixed point, which is obtained from this flow.

One can classify the models of quantum gravity into two classes. The first class is the quantum general relativity, where the renormalization group equation for the Newton constant $G$ does not exist at the perturbative level, at least without the cosmological constant term. In the presence of the cosmological constant there is a perturbative renormalization group equation for the Newton constant, but it is strongly gauge-fixing dependent, as discussed e.g., in [7] and more recently, with very general calculations, in [67] (see more references therein). Let us add that in the functional renormalization group approach the renormalization group flow for $G$ is extracted from the quadratic divergences, and for this reason the corresponding results do not have the perturbative limit. On the other hand, the quadratic divergences are also gauge-fixing dependent [67] and, furthermore, suffer from the total ambiguity in defining the cutoff parameter $\Omega$. For instance, if we start from the total expression for the one-loop divergences in the Schwinger-DeWitt formalism,

$\Gamma_{\text {div }}^{(1)}=-\frac{1}{2} \int d^{4} x \sqrt{g}\left\{\frac{1}{2} A_{0} \Omega^{4}+A_{1} \Omega^{2}+A_{2} \log \left(\frac{\Omega^{2}}{\mu^{2}}\right)\right\}$,

where $A_{0}, A_{1}$, and $A_{2}$ are the algebraic sums of the contributions of quantum metric and ghosts, given by the functional traces of the coincidence limits of the corresponding Schwinger-DeWitt coefficients. Without the cosmological constant only $A_{1}$ has the term linear in the scalar curvature $R$, and hence only this term can contribute to the renormalization group flow for $G$. Now, changing the cutoff as

$$
\Omega^{2} \rightarrow \Omega^{2}+\alpha R,
$$

with an arbitrary coefficient $\alpha$, we observe that the quartic and logarithmic divergences do not change, while the quadratic ones modify according to ${ }^{1}$

$$
A_{1} \rightarrow A_{1}+\alpha A_{0} R
$$

\footnotetext{
${ }^{1}$ One of the authors (I.S.) is grateful to M. Asorey for explaining this point.
}

making it completely ambiguous. Thus, in our opinion, it is difficult to take the results extracted from $A_{1}$ as a physical prediction, even without gauge-fixing ambiguity.

The theories of quantum gravity of the second kind are those with higher derivatives, being fourth derivative [4] models, the super-renormalizable models with six or more derivatives [18], or the nonlocal super-renormalizable models. In all these cases the renormalization group flow in the functional renormalization group has a well-defined perturbative limit (see e.g., [3]), and hence it should be supposed that the invariance or noninvariance of the renormalization group flow should be the same in both usual perturbative and functional renormalization group approaches. On the other hand, without the on-shell invariance condition there is absolutely no way to consider the renormalization group flows in any one of these models in a consistent way. This issue was analyzed in detail in $[7,18,68,69]$ and [19], thus there is no point to repeat these considerations here. Thus, we can conclude that in those versions of functional renormalization group where one can expect a minimally reasonable interpretation of the application to quantum gravity, it is difficult to expect the gauge-invariant flow in the framework of the functional renormalization group, if this approach is not reformulated in a new invariant way.

Finally, we mention two recent papers. In [70] it was shown that the renormalization group flow in quantum general relativity should be compatible with the Ward identities related to the possibility of changing the conformal frame. Certainly, this is not the symmetry that we discussed in the previous sections. Also, even if the mentioned compatibility is achieved, this does not make the whole approach sufficiently unambiguous, as we explained above. However, qualitatively, the importance of the Ward identities to the consistency of the renormalization group flow is certainly coherent with our analysis. On the other hand, Ref. [71] proposed a new formulation of the functional renormalization group for quantum gravity. In this new version, there is not one scale parameter, but two: one for regularizing the UV sector and another one for regularizing the IR sector. The idea is certainly interesting for quantum gravity since the two limits in all known models are very much different. However, since the UV part is essentially the same as in the standard formulation, it is difficult to expect that the gauge-fixing dependence on shell in this new formulation will be a smaller problem than it is in the standard versions with one scale parameter. We can state that the preliminary analysis of this model confirms this qualitative conclusion. The full consideration is possible but would be quite involved and is beyond the scope of the present work.

The results of the considerations which we described above make more interesting the discussion of the possible ways to solve the problem of on-shell gauge-fixing dependence, such as the ones suggested in [34-36] or in [15]. In our opinion, the last approach is more transparent and physically motivated, but (as we have already 
mentioned above) there is no well-developed technique of using it for practical calculations.

\section{ACKNOWLEDGMENTS}

E. A. R. and V.F.B. are grateful to Coordenação de Aperfeiçoamento de Pessoal de Nível Superior (CAPES) for supporting their Ph.D. and Ms. projects. P.M. L. is grateful to the Department of Physics of the Federal University of Juiz de Fora (MG, Brazil) for warm hospitality during his long-term visit, when this work was initiated. The work of P. M. L. was partially supported by the Ministry of Science and Higher Education of the Russian Federation, Grant No. 3.1386.2017 and by the RFBR Grant No. 18-0200153. I. L. S. was partially supported by Conselho Nacional de Desenvolvimento Científico e Tecnológico (CNPq) under the Grant No. 303635/2018-5 and Fundação de Amparo à Pesquisa de Minas Gerais (FAPEMIG) under the Project No. APQ-01205-16.
[1] S. Weinberg, Ultraviolet divergences in quantum theories of gravitation, in General Relativity: An Einstein Centenary Survey, edited by S. W. Hawking and W. Israel (Cambridge University Press, Cambridge, England, 1979).

[2] M. Niedermaier and M. Reuter, The asymptotic safety scenario in quantum gravity, Living Rev. Relativity 9, 5 (2006).

[3] R. Percacci, in Asymptotic Safety, edited by D. Oriti, Approaches to Quantum Gravity (Cambridge University Press, Cambridge, England, 2007), pp. 111-128.

[4] K. S. Stelle, Renormalization of higher derivative quantum gravity, Phys. Rev. D 16, 953 (1977).

[5] F. de O. Salles and I. L. Shapiro, Do we have unitary and (super)renormalizable quantum gravity below the Planck scale?, Phys. Rev. D 89, 084054 (2014); Erratum, Phys. Rev. D 90, 129903 (2014); A. M. Pelinson, F. de Oliveira Salles, and I. L. Shapiro, Gravitational waves and perspectives for quantum gravity, Mod. Phys. Lett. A 29, 1430034 (2014); P. Peter, F. de Oliveira Salles, and I. L. Shapiro, On the ghost-induced instability on de Sitter background, Phys. Rev. D 97, 064044 (2018); A. Salvio, Metastability in quadratic gravity, Phys. Rev. D 99, 103507 (2019); S. C. dos Reis, G. Chapiro, and I. L. Shapiro, Beyond the linear analysis of stability in higher derivative gravity with the Bianchi-I metric, Phys. Rev. D 100, 066004 (2019).

[6] A. Salam and J. Strathdee, Remarks on high-energy stability and renormalizability of gravity theory, Phys. Rev. D 18, 4480 (1978).

[7] E. S. Fradkin and A. A. Tseytlin, Renormalizable asymptotically free quantum theory of gravity, Nucl. Phys. B201, 469 (1982).

[8] I. G. Avramidi and A. O. Barvinsky, Asymptotic freedom in higher derivative quantum gravity, Phys. Lett. 159B, 269 (1985).

[9] G. de Berredo-Peixoto and I. L. Shapiro, Higher derivative quantum gravity with Gauss-Bonnet term, Phys. Rev. D 71, 064005 (2005).

[10] E. Tomboulis, 1/N expansion and renormalization in quantum gravity, Phys. Lett. 70B, 361 (1977); Renormalizability and asymptotic freedom in quantum gravity, Phys. Lett. 97B, 77 (1980); Unitarity in Higher Derivative Quantum Gravity, Phys. Rev. Lett. 52, 1173 (1984).
[11] I. Antoniadis and E. T. Tomboulis, Gauge invariance and unitarity in higher derivative quantum gravity, Phys. Rev. D 33, 2756 (1986).

[12] D. A. Johnston, Sedentary ghost poles in higher derivative gravity, Nucl. Phys. B297, 721 (1988).

[13] A. Codello and R. Percacci, Fixed Points of Higher Derivative Gravity, Phys. Rev. Lett. 97, 221301 (2006); A. Codello, R. Percacci, L. Rachwal, and A. Tonero, Computing the effective action with the functional renormalization group, Eur. Phys. J. C 76, 226 (2016).

[14] A. Codello, R. Percacci, and C. Rahmede, Investigating the ultraviolet properties of gravity with a Wilsonian renormalization group equation, Ann. Phys. (Amsterdam) 324, 414 (2009).

[15] P. M. Lavrov and I. L. Shapiro, On the functional renormalization group approach for Yang-Mills fields, J. High Energy Phys. 06 (2013) 086.

[16] P. M. Lavrov, Gauge (in)dependence and background field formalism, Phys. Lett. B 791, 293 (2019).

[17] P. M. Lavrov, E. A. dos Reis, T. de Paula Netto, and I. L. Shapiro, Gauge invariance of the background average effective action, Eur. Phys. J. C 79, 661 (2019).

[18] M. Asorey, J. L. López, and I. L. Shapiro, Some remarks on high derivative quantum gravity, Int. J. Mod. Phys. A 12, 5711 (1997).

[19] L. Modesto, L. Rachwał, and I. L. Shapiro, Renormalization group in superrenormalizable quantum gravity, Eur. Phys. J. C 78, 555 (2018).

[20] C. N. Yang and R. L. Mills, Conservation of isotopic spin and isotopic gauge invariance, Phys. Rev. 96, 191 (1954).

[21] R. Jackiw, Functional evaluation of the effective potential, Phys. Rev. D 9, 1686 (1974).

[22] L. Dolan and R. Jackiw, Gauge invariant signal for gauge symmetry breaking, Phys. Rev. D 9, 2904 (1974).

[23] N. K. Nielsen, On the gauge dependence of spontaneous symmetry breaking in gauge theories, Nucl. Phys. B101, 173 (1975).

[24] R. Fukuda and T. Kugo, Gauge invariance in the effective action and potential, Phys. Rev. D 13, 3469 (1976).

[25] P. M. Lavrov and I. V. Tyutin, On the generating functional for the vertex functions in Yang-Mills theories, Yad. Fiz. 34, 277 (1981). 
[26] B. L. Voronov, P. M. Lavrov, and I. V. Tyutin, Canonical transformations and gauge dependence in general gauge theories, Yad. Fiz. 36, 498 (1962).

[27] C. Wetterich, Average action and the renormalization group equations, Nucl. Phys. B B352, 529 (1991).

[28] C. Wetterich, Exact evolution equation for the effective potential, Phys. Lett. B 301, 90 (1993).

[29] J. Berges, N. Tetradis, and C. Wetterich, Nonperturbative renormalization flow in quantum field theory and statistical physics, Phys. Rep. 363, 223 (2002).

[30] C. Bagnuls and C. Berviller, Exact renormalization group equations: An introductory review, Phys. Rep. 348, 91 (2001).

[31] J. Polonyi, Lectures on the functional renormalization group method, Central Eur. J. Phys. 1, 1 (2003).

[32] J. M. Pawlowski, Aspects of the functional renormalization group, Ann. Phys. (Amsterdam) 322, 2831 (2007).

[33] B. Delamotte, An introduction to the nonperturbative renormalization group, Lect. Notes Phys. 852, 49 (2012).

[34] O. J. Rosten, Fundamentals of the exact renormalization group, Phys. Rep. 511, 177 (2012).

[35] T. R. Morris, A gauge invariant exact renormalization group. 2, J. High Energy Phys. 12 (2000) 012.

[36] S. Arnone, T. R. Morris, and O. J. Rosten, A generalized manifestly gauge invariant exact renormalization group for SU(N) Yang-Mills, Eur. Phys. J. C 50, 467 (2007).

[37] V. Branchina, K. A. Meissner, and G. Veneziano, The prize of an exact, gauge invariant RG flow equation, Phys. Lett. B 574, 319 (2003).

[38] J. M. Pawlowski, Geometrical effective action and Wilsonian flows, arXiv:hep-th/0310018.

[39] G. A. Vilkovisky, in B.S. DeWitt Sixtieth Anniversary Volume, edited by S. Christensen (Hilger, Bristol, United Kingdom, 1983).

[40] G. A. Vilkovisky, The unique effective action in quantum field theory, Nucl. Phys. B234, 125 (1984).

[41] B. S. DeWitt, Quantum theory of gravity. II. The manifestly covariant theory, Phys. Rev. 162, 1195 (1967).

[42] I. Ya. Arefeva, L. D. Faddeev, and A. A. Slavnov, Generating functional for the s matrix in gauge theories, Teor. Mat. Fiz. 21, 311 (1975) [Theor. Math. Phys. 21, 1165 (1975)].

[43] L. F. Abbott, The background field method beyond one loop, Nucl. Phys. B185, 189 (1981).

[44] A. O. Barvinsky, D. Blas, M. Herrero-Valca, S. M. Sibiryakov, and C. F. Steinwachs, Renormalization of gauge theories in the background-field approach, J. High Energy Phys. 07 (2018) 035 .

[45] I. A. Batalin, P. M. Lavrov, and I. V. Tyutin, Multiplicative renormalization of Yang-Mills theories in the backgroundfield formalism, Eur. Phys. J. C 78, 570 (2018).

[46] J. Frenkel and J. C. Taylor, Background gauge renormalization and BRST identities, Ann. Phys. (Amsterdam) 389, 234 (2018).

[47] I. A. Batalin, P. M. Lavrov, and I. V. Tyutin, Gauge dependence and multiplicative renormalization of Yang-Mills theory with matter fields, Eur. Phys. J. C 79, 628 (2019).

[48] P. M. Lavrov and I. L. Shapiro, Gauge invariant renormalizability of quantum gravity, Phys. Rev. D 100, 026018 (2019).

[49] B. L. Giacchini, P. M. Lavrov, and I. L. Shapiro, Background field method and nonlinear gauges, Phys. Lett. B 797, 134882 (2019).
[50] R. Percacci and G. P. Vacca, The background scale Ward identity in quantum gravity, Eur. Phys. J. C 77, 52 (2017).

[51] G. 't Hooft, An algorithm for poles at dimension four in the dimensional regularization procedure, Nucl. Phys. B62, 444 (1973).

[52] H. Klusberg-Stern and J. B. Zuber, Renormalization of nonAbelian gauge theories in a background-field gauge. I. Green's functions, Phys. Rev. D 12, 482 (1975).

[53] M. T. Grisaru, P. van Nieuwenhuizen, and C. C. Wu, Background-field method versus normal field theory in explicit examples: One-loop divergences in the $S$ matrix and Green's functions for Yang-Mills and gravitational fields, Phys. Rev. D 12, 3203 (1975).

[54] D. M. Capper and A. MacLean, The background field method at two loops: A general gauge Yang-Mills calculation, Nucl. Phys. B203, 413 (1982).

[55] S. Ichinose and M. Omote, Renormalization using the background-field formalism, Nucl. Phys. B203, 221 (1982).

[56] M. H. Goroff and A. Sagnotti, The ultraviolet behavior of Einstein gravity, Nucl. Phys. B266, 709 (1986).

[57] A. E. M. van de Ven, Two-loop quantum gravity, Nucl. Phys. B378, 309 (1992).

[58] P. A. Grassi, Algebraic renormalization of Yang-Mills theory with background field method, Nucl. Phys. B462, 524 (1996).

[59] C. Becchi and R. Collina, Further comments on the background field method and gauge invariant effective action, Nucl. Phys. B562, 412 (1999).

[60] R. Ferrari, M. Picariello, and A. Quadri, Algebraic aspects of the background field method, Ann. Phys. (N.Y.) 294, 165 (2001).

[61] B. S. DeWitt, Dynamical Theory of Groups and Fields (Gordon and Breach, New York, 1965).

[62] L. D. Faddeev and V. N. Popov, Feynman diagrams for the Yang-Mills field, Phys. Lett. 25B, 29 (1967).

[63] BC. Becchi, A. Rouet, and R. Stoura, The Abelian Higgs Kibble model, unitarity of the S-operator, Phys. Lett. 52B, 344 (1974).

[64] I. V. Tyutin, Gauge invariance in field theory and statistical physics in operator formalism, arXiv:0812.0580.

[65] R. Delbourgo and M. Ramon-Medrano, Becchi-Rouet-Stora gauge identities for gravity, Nucl. Phys. B110, 467 (1976).

[66] P. K. Townsend and P. van Nieuwenhuizen, BRS gauge and ghost field supersymmetry in gravity and supergravity, Nucl. Phys. B120, 301 (1977).

[67] J. D. Gonçalves, T. de Paula Netto, and I. L. Shapiro, On the gauge and parametrization ambiguity in quantum gravity, Phys. Rev. D 97, 026015 (2018).

[68] I. G. Avramidi, Covariant methods for the calculation of the effective action in quantum field theory and investigation of higher-derivative quantum gravity, Ph.D. Thesis, Moscow University, 1986.

[69] I. L. Shapiro and A. G. Jacksenaev, Gauge dependence in higher derivative quantum gravity and the conformal anomaly problem, Phys. Lett. B 324, 286 (1994).

[70] P. Labus, T. R. Morris, and Z. H. Slade, Background independence in a background dependent renormalization group, Phys. Rev. D 94, 024007 (2016).

[71] S.P. de Alwis, Exact RG flow equations and quantum gravity, J. High Energy Phys. 03 (2018) 118. 